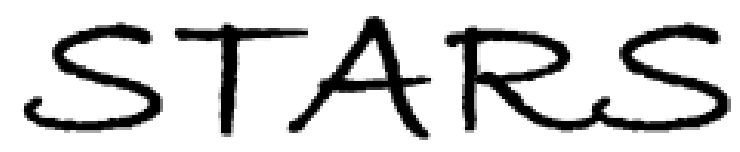

University of Central Florida

STARS

$1-1-2009$

\title{
Diffusion under temperature gradient: A phase-field model study
}

\author{
R. R. Mohanty \\ University of Central Florida \\ J. E. Guyer \\ Y. H. Sohn \\ University of Central Florida
}

Find similar works at: https://stars.library.ucf.edu/facultybib2000

University of Central Florida Libraries http://library.ucf.edu

This Article is brought to you for free and open access by the Faculty Bibliography at STARS. It has been accepted for inclusion in Faculty Bibliography 2000 s by an authorized administrator of STARS. For more information, please contactSTARS@ucf.edu.

\section{Recommended Citation}

Mohanty, R. R.; Guyer, J. E.; and Sohn, Y. H., "Diffusion under temperature gradient: A phase-field model study" (2009). Faculty Bibliography 2000s. 1917.

https://stars.library.ucf.edu/facultybib2000/1917

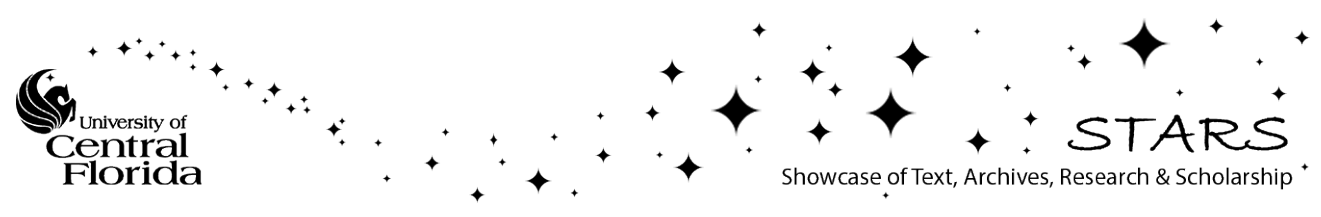




\section{Diffusion under temperature gradient: A phase-field model study}

Cite as: J. Appl. Phys. 106, 034912 (2009); https://doi.org/10.1063/1.3190607

Submitted: 27 February 2009 . Accepted: 04 July 2009 . Published Online: 11 August 2009

R. R. Mohanty, J. E. Guyer, and Y. H. Sohn

\section{ARTICLES YOU MAY BE INTERESTED IN}

Free Energy of a Nonuniform System. I. Interfacial Free Energy

The Journal of Chemical Physics 28, 258 (1958); https://doi.org/10.1063/1.1744102

Models for numerical treatment of multicomponent diffusion in simple phases

Journal of Applied Physics 72, 1350 (1992); https://doi.org/10.1063/1.351745

Phase Separation by Spinodal Decomposition in Isotropic Systems

The Journal of Chemical Physics 42, 93 (1965); https://doi.org/10.1063/1.1695731

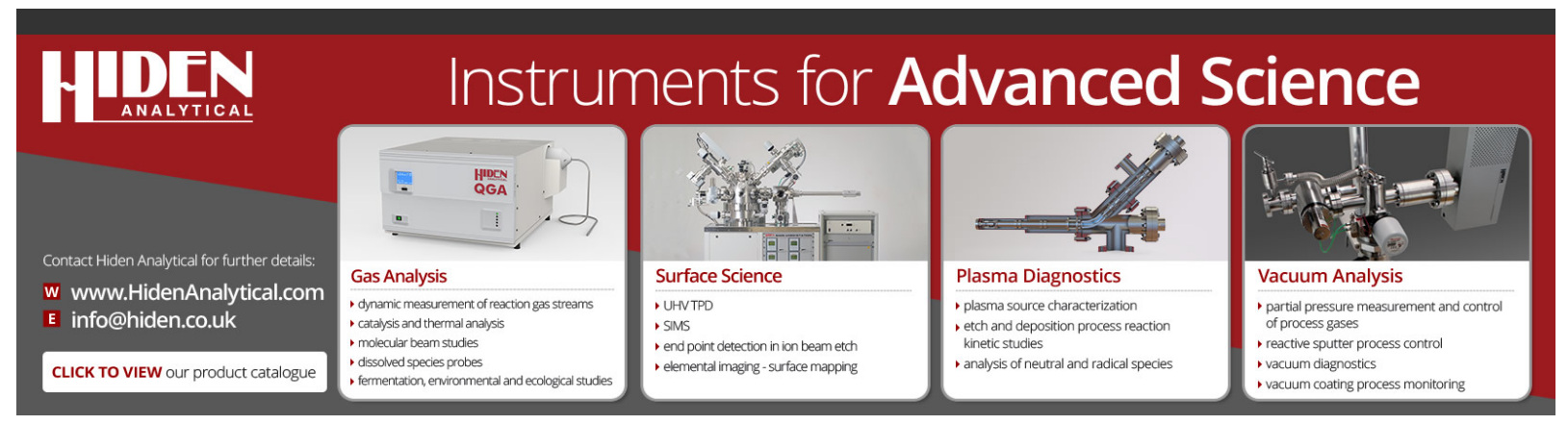




\title{
Diffusion under temperature gradient: A phase-field model study
}

\author{
R. R. Mohanty, ${ }^{1, a)}$ J. E. Guyer, ${ }^{2, b)}$ and Y. H. Sohn ${ }^{1, c)}$ \\ ${ }^{1}$ Department of Mechanical, Materials and Aerospace Engineering and Advanced Materials Processing and \\ Analysis Center, 4000 Central Florida Blvd., University of Central Florida, Orlando, Florida 32816, \\ USA \\ ${ }^{2}$ Metallurgy Division, National Institute of Standards and Technology (NIST), 100 Bureau Drive, Stop 8554, \\ Gaithersburg, Maryland 20899, USA
}

(Received 27 February 2009; accepted 4 July 2009; published online 11 August 2009)

A diffuse interface model was devised and employed to investigate the effect of thermotransport (a.k.a., thermomigration) process in single-phase and two-phase alloys of a binary system. Simulation results show that an applied temperature gradient can cause significant redistribution of constituent elements and phases in the alloy. The magnitude and the direction of the redistribution depend on the initial composition, the atomic mobility and the heat of transport of the respective elements. In two-phase alloys, the thermomigration effect can cause the formation of single-element rich phases at the cold and hot ends of the alloy (i.e., demixing). (C) 2009 American Institute of Physics. [DOI: 10.1063/1.3190607]

\section{INTRODUCTION}

It is well known that when a temperature gradient is applied to a homogeneous alloy of more than one component, a concentration gradient can develop and eventually reach a steady state, with the concentration gradient being a characteristic of the system. ${ }^{1}$ This phenomenon is known as thermomigration or thermotransport or Soret or LudwigSoret effect. The thermomigration effect can play an important role in microstructural stability, for example, in interconnects of electronic circuits, metallic nuclear fuel alloys, superalloys, and coatings used in gas turbine engines, etc., where a significant temperature gradient is imposed, owing to increasing operating temperatures and/or reducing length scales of these systems and/or ingenuity in internal cooling methods. Depending upon applications, temperature gradient imposed for a prolonged period of time can vary from $20{ }^{\circ} \mathrm{C} / \mathrm{mm}$ up to $1500{ }^{\circ} \mathrm{C} / \mathrm{mm}$ although its magnitude can be reduced by means of various engineering solutions (e.g., thermal barrier coatings). Nevertheless, the temperature gradient imposed can certainly induce thermotransport of constitutent elements and phases, and alter the microstructure of materials carefully designed for specific engineering applications.

Thermomigration can produce significant redistribution of elements and constituent phases in single- and two-phase alloys. This redistribution can induce many unwanted changes such as melting, phase transformations, and other changes in physical and mechanical properties, all of which degrade the stability and performance of components. Examples of the thermomigration effect can be found in literature published over the past 5 decades. ${ }^{2-14}$

There are two important parameters in the thermomigra-

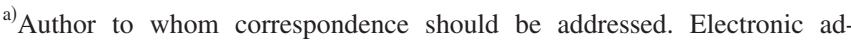
dresses: rmohanty@mail.ucf.edu and mohantyrr@gmail.com. Present address: ArcelorMittal Global R\&D, East Chicago, Indiana 46312.

${ }^{b)}$ Electronic mail: guyer@nist.gov.

${ }^{c)}$ Electronic mail: ysohn@mail.ucf.edu.
}

tion study: the mobility or diffusivity of atoms present, and their heat of transport $\left(Q_{i}^{*}\right)$ that is related to the amount of heat carried per atom of species $i .^{3}$ In general, the value of $Q_{i}^{*}$ is the contribution of the flux of species $i$ to the flux of heat, and determines the affinity of a species toward the cold or hot end. When the solute diffusivity is much higher than the solvent, e.g., interstitial solute, only one heat of transport term is sufficient to describe the flux under a temperature gradient. However, as explained by Jaffe and Shewmon ${ }^{5}$ both mobility and heat of transport terms of individual elements are required to accurately describe the atom flux in a substitutional alloy subjected to a temperature gradient, even when both elements have similar mobilities. In such cases, the complexity of the problem often necessitates the use of computational modeling to understand and predict the thermomigration behavior in alloys.

Tikare and Holm ${ }^{15}$ used a Potts Monte Carlo technique to simulate grain growth and pore migration in a thermal gradient, where they utilized the atomic interaction energy in the model. In another study, Snyder et al. ${ }^{16}$ simulated Ostwald ripening under the influence of temperature gradient, where they neglected the Soret effect and considered only the mass flow due to the temperature dependence of the local equilibrium interfacial composition. There are other computational models ${ }^{17-21}$ reported that described the thermomigration problem in one dimension with the following assumptions: (1) transport of the solute takes place only in the matrix phase; (2) second phase particles act as sources or sinks for the solute atoms in solid solution; (3) solute diffusivity is much faster than the solvent; and (4) local thermodynamic equilibrium exists at the boundary between the two phases. Such a priori assumptions require that the positions of the boundary between the phases be known, which makes the numerical solution difficult to achieve. Unlike these models, a diffuse interface (Cahn-Hilliard) model ${ }^{22}$ does not require the above assumptions to be made and eliminates the requirement of tracking the boundary by treating the interface as diffused over a certain width. ${ }^{23}$ In addition, a diffuse 
interface model can be derived phenomenologically and material specific thermodynamic and kinetic data can be employed as input parameters.

In this paper we report the development of a diffuse interface model and computer simulation study of time evolution in composition profiles and microstructure in single and two-phase alloys of a binary system subjected to a temperature gradient. A simple regular solution model with various combinations of atomic mobility and heat of transport terms were used in this study.

\section{PHASE FIELD MODEL FOR THERMOTRANSPORT IN BINARY ALLOYS}

\section{A. Phenomenological formulation}

We consider a binary substitutional alloy of elements $A$ and $B$, whose molar volume $\left(V_{m}\right)$ and density $(\rho)$ are assumed to be constant. Let $n_{i}$ and $c_{i}$ represent the concentration and mole fraction of an element $i$, respectively, so that

$$
\sum_{i} n_{i}=\rho=\frac{1}{V_{m}} \text { and } \quad \sum_{i} c_{i}=1.0 .
$$

Flux of an element under the gradients of concentration and temperature can be defined by the following equation given by de Groot $^{24}$

$$
J_{k}=\sum_{i=1}^{n-1} L_{k i}\left(X_{i}-X_{n}+Q_{i}^{*} X_{q}\right)
$$

where $n$ is the total number of elements, $Q_{i}^{*}$ is the heat of transport term, and $X$ stands for the driving forces such as

$$
X_{i}=-\left(\nabla \mu_{i}\right)_{T} \quad \text { and } \quad X_{q}=-\frac{\nabla T}{T} .
$$

Note that Eq. (2) is obtained by the linear transformation and the gradient in chemical potential, i.e., $\left(\nabla \mu_{i}\right)_{T}$ is due to gradients in concentration, but not temperature. ${ }^{24-26}$

Howard and Lidiard ${ }^{25}$ applied the above equation to describe thermotransport on the basis of a vacancy mechanism in a binary substitutional alloy. The general flux equations for an alloy of elements $A$ and $B$ are expressed by

$$
J_{A}=L_{A A}\left(X_{A}-X_{v}+Q_{A}^{*} X_{q}\right)+L_{A B}\left(X_{B}-X_{v}+Q_{B}^{*} X_{q}\right),
$$

and

$$
J_{B}=L_{B A}\left(X_{A}-X_{v}+Q_{A}^{*} X_{q}\right)+L_{B B}\left(X_{B}-X_{v}+Q_{B}^{*} X_{q}\right),
$$

where $v$ corresponds to vacancies. Again following Howard and Lidiard, ${ }^{25}$ assuming that vacancies exist everywhere in their equilibrium concentration, $\nabla \mu_{v}=0$, but in a temperature gradient $\left(\nabla \mu_{v}\right)_{T} \neq 0$. Hence

$$
X_{v}=-\left(\nabla \mu_{v}\right)_{T}=-k T \frac{\nabla c_{v}}{c_{v}}=-h_{f v} \frac{\nabla T}{T}=h_{f v} X_{q},
$$

where $h_{f v}$ is the enthalpy of vacancy formation. Substituting Eq. (5) in Eq. (4) yields

$$
J_{A}=L_{A A}\left[X_{A}+\left(Q_{A}^{*}-h_{f v}\right) X_{q}\right]+L_{A B}\left[X_{B}+\left(Q_{B}^{*}-h_{f v}\right) X_{q}\right]
$$

and

$$
J_{B}=L_{B A}\left[X_{A}+\left(Q_{A}^{*}-h_{f v}\right) X_{q}\right]+L_{B B}\left[X_{B}+\left(Q_{B}^{*}-h_{f v}\right) X_{q}\right] .
$$

From Prigogine's theorem, ${ }^{26,27}$ which is a re-expression of the Gibbs-Duhem equation, it follows:

$$
c_{A} X_{A}+c_{B} X_{B}+c_{v} X_{v}=0 .
$$

Further, assuming $c_{v} \ll c_{A}, c_{B}$,

$$
c_{A} X_{A}+c_{B} X_{B} \approx 0 \text {. }
$$

Applying Eq. (7b) and the condition for conservation of mass given in Eq. (1), one gets

$$
L_{A A} X_{A}+L_{A B} X_{B}=\frac{c L_{A A}-(1-c) L_{A B}}{c} X_{A}=\rho(1-c) \beta_{A} X_{A} \text {, }
$$

and

$$
L_{B B} X_{B}+L_{B A} X_{A}=\frac{(1-c) L_{B B}-c L_{B A}}{1-c} X_{B}=\rho c \beta_{B} X_{B}
$$

where $c$ is the composition of $B$ and $\beta_{i}$ is the atomic mobility of element $i$. The general flux equation under isothermal conditions, i.e., $J_{i}=-n_{i} \beta_{i} X_{i}$ has been used to derive Eq. (8). Now substituting Eq. (8) into flux equations, Eq. (6) yields

$$
\begin{aligned}
J_{A}= & -\rho(1-c) \beta_{A} \nabla \mu_{A} \\
& +\left[L_{A A}\left(Q_{A}^{*}-h_{f v}\right)+L_{A B}\left(Q_{B}^{*}-h_{f v}\right)\right] X_{q},
\end{aligned}
$$

and

$$
J_{B}=-\rho c \beta_{B} \nabla \mu_{B}+\left[L_{B A}\left(Q_{A}^{*}-h_{f v}\right)+L_{B B}\left(Q_{B}^{*}-h_{f v}\right)\right] X_{q} .
$$

A vacancy mechanism being operative, it is convenient to choose a laboratory frame of reference where the sum of the fluxes for two elements vanishes, ${ }^{28}$ such that

$$
\widetilde{J}_{A}+\widetilde{J}_{B}=0
$$

and

$$
\widetilde{J}_{A}=-\tilde{J}_{B}=J_{A}-(1-c)\left(J_{A}+J_{B}\right)=c J_{A}-(1-c) J_{B} .
$$

Substituting respective intrinsic fluxes from Eq. (9) in Eq. (11) yields

$$
\begin{aligned}
\tilde{J}_{A}= & -\rho c(1-c) \beta_{A} \nabla \mu_{A}+\rho c(1-c) \beta_{B} \nabla \mu_{B} \\
& +c\left[L_{A A}\left(Q_{A}^{*}-h_{f v}\right)+L_{A B}\left(Q_{B}^{*}-h_{f v}\right)\right] X_{q} \\
& -(1-c)\left[L_{B A}\left(Q_{A}^{*}-h_{f v}\right)+L_{B B}\left(Q_{B}^{*}-h_{f v}\right)\right] X_{q} .
\end{aligned}
$$

Using Eq. (8) along with the Onsager's reciprocal relationship $L_{i j}=L_{j i}$, 


$$
\begin{aligned}
\widetilde{J}_{A}= & -\rho c(1-c) \beta_{A} \nabla \mu_{A}+\rho c(1-c) \beta_{B} \nabla \mu_{B} \\
& +\left[c L_{A A}-(1-c) L_{B A}\right] \widetilde{Q}_{A}^{*} X_{q} \\
& +\left[c L_{A B}-(1-c) L_{B B}\right] \widetilde{Q}_{B}^{*} X_{q} \\
= & -\rho c(1-c) \beta_{A} \nabla \mu_{A}+\rho c(1-c) \beta_{B} \nabla \mu_{B} \\
& +\left[\rho c(1-c) \beta_{A} \widetilde{Q}_{A}^{*}-\rho c(1-c) \beta_{B} \widetilde{Q}_{B}^{*}\right] X_{q},
\end{aligned}
$$

where

$$
\widetilde{Q}_{i}^{*}=Q_{i}^{*}-h_{f v} .
$$

Again using the Gibbs-Duhem equation, $(1-c) \nabla \mu_{A}$ $+c \nabla \mu_{B}=0$, one gets

$$
\nabla \mu_{A}=c \nabla \mu_{A}^{\text {eff }}, \nabla \mu_{B}=-(1-c) \nabla \mu_{A}^{\text {eff }},
$$

where

$$
\nabla \mu_{A}^{\text {eff }}=-\nabla \mu_{B}^{\text {eff }}=\nabla\left(\mu_{A}-\mu_{B}\right) .
$$

Now substituting Eq. (15) into Eq. (12) yields

$$
\begin{aligned}
\widetilde{J}_{A}= & -\rho c(1-c)\left[c \beta_{A}+(1-c) \beta_{B}\right] \nabla \mu_{A}^{\mathrm{eff}} \\
& +\rho c(1-c)\left[\beta_{A} \widetilde{Q}_{A}^{*}-\beta_{B} \widetilde{Q}_{B}^{*}\right] X_{q} \\
= & -M_{c} \nabla \mu_{A}^{\mathrm{eff}}+M_{Q} X_{q}=M_{c} \nabla \mu_{B}^{\mathrm{eff}}-M_{Q} \frac{\nabla T}{T},
\end{aligned}
$$

and

$$
\widetilde{J}_{B}=-\widetilde{J}_{A}=-M_{c} \nabla \mu_{B}^{\mathrm{eff}}+M_{Q} \frac{\nabla T}{T},
$$

where the chemical mobility is defined as

$$
M_{c}=\rho c(1-c)\left[c \beta_{A}+(1-c) \beta_{B}\right],
$$

and the heat of transport term, that can be referred to as "mobility of thermotransport," is defined as

$$
M_{Q}=\rho c(1-c)\left[\beta_{A} \widetilde{Q}_{A}^{*}-\beta_{B} \widetilde{Q}_{B}^{*}\right] .
$$

In Eq. (17), the effective chemical potential for a nonhomogeneous system is defined by

$$
\mu_{B}^{\text {eff }}=\frac{\delta F}{\delta n_{B}}=V_{m} \frac{\delta F}{\delta c},
$$

where $\delta$ stands for the variational derivative, and $F$ is the Cahn-Hilliard free energy functional consisting of the bulk free energy and interfacial energy contributions, expressed by

$$
F=F_{\text {bulk }}+F_{\text {int }}=N_{V} \int_{V}\left[f(c, T)+\kappa(\nabla c)^{2}\right] d V .
$$

Here, $f(c, T)$ is the Helmholtz free energy density, approximated by the regular solution model, and $\kappa$ is the gradient energy coefficient associated with the gradient of composition. Note that $f(c, T)$ can be directly adopted from the thermodynamic databases if available for a particular alloy system. Using Eqs. (21) and (22) in Eq. (18), the flux equation becomes

$$
\widetilde{J}_{B}=-V_{m} M_{c} \nabla\left(\frac{\partial f}{\partial c}-2 \kappa_{c} \nabla^{2} c\right)+M_{Q} \frac{\nabla T}{T} .
$$

The spatiotemporal evolution of the composition can now be expressed by the continuity equation

$$
\begin{aligned}
\frac{\partial n_{B}}{\partial t} & =\frac{1}{V_{m}} \frac{\partial c(x, t)}{\partial t}=-\nabla \cdot \widetilde{J}_{B} \\
& =\nabla \cdot\left[V_{m} M_{c} \nabla\left(\frac{\partial f}{\partial c}-2 \kappa_{c} \nabla^{2} c\right)-M_{Q} \frac{\nabla T}{T}\right] .
\end{aligned}
$$

Notice that temperature-dependent or constant atomic mobility and heat of transport values can be used in the above equation. For the simulation of single-phase alloys we have employed constant atomic mobility and heat of transport terms for simpler description of the thermotransport phenomena. For two-phase alloys, we have used the Arrhenius and linear relations to express the temperature dependency of atomic mobility and heat of transport terms, respectively, ${ }^{9,29,30}$ as $\beta_{i}=\beta_{0} \exp \left(-Q_{i} / R T\right)$ and $\widetilde{Q}_{i}^{*}=C_{i}+D_{i} T$ where $i=A, B$.

\section{B. Numerical procedure}

For numerical convenience, the governing Eq. (24) was rewritten in the following dimensionless form:

$$
\begin{aligned}
\frac{\partial \bar{c}(\mathbf{x}, \tau)}{\partial \tau}= & \bar{\nabla}\left[\bar{M}_{c}(\bar{c}) \bar{\nabla}\left(\frac{\partial \bar{f}}{\partial \bar{c}(\mathbf{x}, \tau)}-\bar{\kappa} \bar{\nabla} \bar{c}(\mathbf{x}, \tau)\right)\right. \\
& \left.-\bar{M}_{Q} \frac{\bar{\nabla} T}{T}\right],
\end{aligned}
$$

by introducing the following reduced quantities of

$$
\bar{\nabla}=\left(\frac{\partial}{\partial(x / l)}, \frac{\partial}{\partial(y / l)}\right),
$$

$\bar{M}_{c}=V_{m} M_{c} / \beta, \bar{f}=V_{m} f / \Delta f, \bar{\kappa}=\kappa V_{m} / \Delta f l^{2}, \bar{M}_{Q}=M_{Q} V_{m} / \Delta f \beta$, and $\tau=\beta \Delta f / l^{2} t$, where $\mathbf{x}$ and $\tau$ are the reduced length and time, $l$ is the length scale of the system, $\Delta f\left(=R T_{c}\right)$ is the normalization factor for the free energy with $T_{c}=900 \mathrm{~K}, R$ is the universal gas constant, $V_{m}$ is the constant molar volume, and $\beta$ is a constant of atomic mobility. The above equation with appropriate boundary conditions was solved numerically using a control volume method implemented by the FiPy partial differential equation solver. ${ }^{31}$ Boundary conditions for both the 1D and 2D cases can be expressed as

$$
\hat{n} \cdot \nabla \bar{c}=0 \quad \text { and } \hat{n} \cdot \nabla^{3} \bar{c}=0
$$

on all exterior boundaries.

The applied temperature field obeys Laplace's equation expressed by

$$
\nabla^{2} T=0
$$

with boundary conditions described as

$$
J_{q} \cdot \hat{n}=0,\left.T\right|_{x=0}=T_{\min },\left.T\right|_{x=L}=T_{\max } .
$$

The above conditions produce a linear distribution of temperature across the system. Note that the equation for heat 


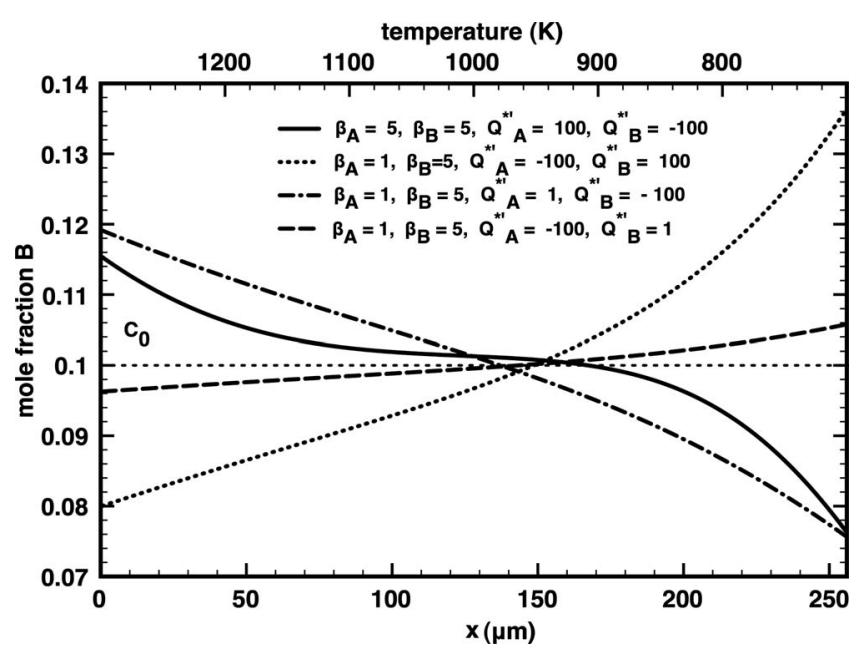

FIG. 1. Composition profiles developed in an initially homogeneous $\left(c_{0}\right.$ $=0.1$ ) single-phase alloy after being subjected to a temperature gradient for $6 \mathrm{~h}$. Temperature ranged from $T_{\max }=1273 \mathrm{~K}$ on the left end and $T_{\min }$ $=773 \mathrm{~K}$ on the right end of the system.

flux is not considered here as the heat flux due to mass flux, i.e., the Dufour effect is considered negligible and ignored.

\section{RESULTS}

\section{A. Single-phase alloy}

A single-phase alloy with initial homogeneous composition of $c_{0}=0.1$ was subjected to a temperature gradient, where $T_{\max }=1273 \mathrm{~K}$ and $T_{\min }=773 \mathrm{~K}$. The left end $(L=0)$ of the system is the hotter end for this part of the simulation. Different combinations of atomic mobility and heat of transport terms, which are not functions of temperature, were employed and the resulting composition profiles are presented in Fig. 1. The grid resolution was chosen to be $\Delta x=1 \mu \mathrm{m}$ for one-dimensional (1D) calculations, and value of the gradient energy coefficient was set to zero. The results show that upon the application of the temperature gradient, a composition gradient develops in the alloy, where the elements move toward the hot or cold end depending on the magnitude and sign of the mobility and heat of transport terms. The magnitude of the concentration gradient developed in the alloy is also determined by the above two factors. As is observed from the composition profiles, it can be said without any generalization, that the element with a negative heat of transport moves toward the hot end of the system. A steady state can be achieved with prolonged annealing under thermal gradient when the contribution to the flux due to temperature gradient becomes equal to that of concentration gradient. In Fig. 2, a representative composition profile is shown along with the flux profiles as the system nears a steady state. The contributions to the total flux due to the chemical potential gradient and the temperature gradient are almost equal in magnitude but opposite in direction.

\section{B. Two-phase alloy}

The simulation of two-phase alloys was performed by applying a temperature gradient to a pre-generated two-phase microstructure. A representative microstructure of this two-

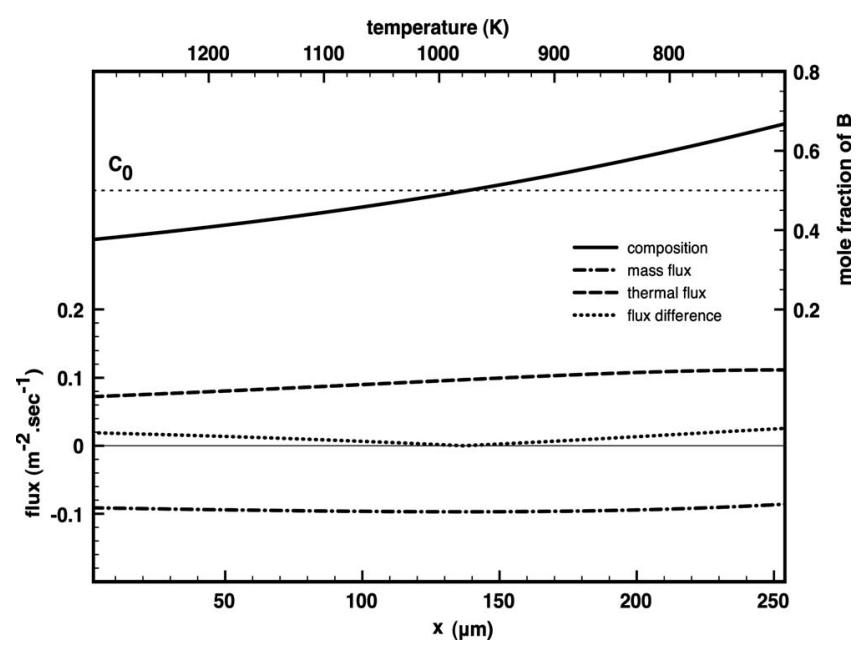

FIG. 2. Representative profiles of composition and flux in an initially homogeneous $\left(c_{0}=0.5\right)$ single-phase alloy approaching steady state after being subjected to annealing in a temperature gradient. Temperature ranged from $T_{\max }=1273 \mathrm{~K}$ on the left end and $T_{\min }=773 \mathrm{~K}$ on the right end of the system. "Mass flux" and "thermal flux" are the contributions of chemical potential gradient and temperature gradient, respectively, to the total flux. The "flux difference" is the difference between these two contributions (i.e., net flux).

phase alloy is presented in Fig. 3(a). The microstructure was generated first by introducing $\beta$ nuclei (second phase) randomly into a $\alpha$ matrix at a temperature of $900 \mathrm{~K}$. This "nucleated" microstructure was annealed isothermally at 900 $\mathrm{K}$ until the phases attend their equilibrium composition prior to simulation of thermotransport. The "annealed" two-phase microstructure served as the initial microstructure/ composition-distribution that was subjected to a constant temperature gradient from $800 \mathrm{~K}$ to $1000 \mathrm{~K}$. The following parameters were chosen: $\Delta x=5 \mu \mathrm{m}$, and $\bar{\kappa}=1.0$.

Before starting the simulation for thermomigration, it is interesting to see the microstructural changes that could happen under a temperature gradient without the thermomigration term being considered in the flux equations. For this purpose, a simulation was performed by applying the aforementioned temperature gradient to a two-phase alloy, while turning the thermomigration term off, i.e., $Q_{A}^{*}=Q_{B}^{*}=0$. The resulting microstructure is presented in Fig. 3(b). While there is no redistribution of phases across the system, there is a difference in the compositions of phases at the hot and cold sides, evidenced by contrasts between phases. This slight changes in composition is due to the variation in equilibrium composition dictated by the thermodynamic description of the system.

Finally, the thermomigration term was "turned-on," and four different combinations of heat of transport values were examined to study their effect on the final microstructure. In all the four cases studied, the same atomic mobilities were used, wherein $\beta_{B}>\beta_{A}$ and $Q_{A}^{*}, Q_{B}^{*}>0$. In case I: $Q_{A}^{*}=Q_{B}^{*}$ and $M_{Q}<0$, in case II: $Q_{B}^{*} \gg Q_{A}^{*}$ and $M_{Q}<0$, in case III: $Q_{B}^{*}$ $\ll Q_{A}^{*}$ and $M_{Q}>0$, and in case IV: $Q_{B}^{*}<Q_{A}^{*}$ and $M_{Q}>0$. The chemical mobility, $M_{c}$ was positive for all the above cases. Microstructures obtained for these four different conditions are presented in Figs. 4-7, respectively. A considerable redistribution of the second phase occurs along with the for- 


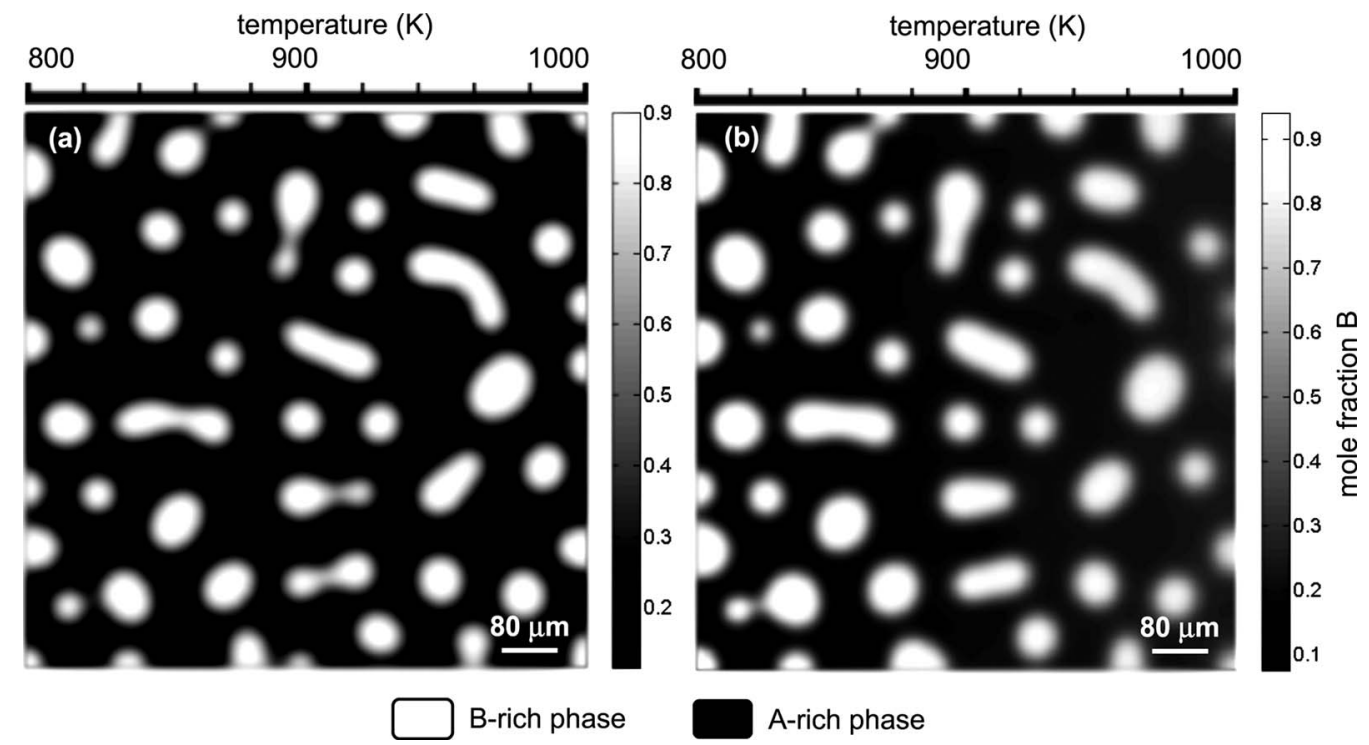

FIG. 3. (a) A representative micrograph of the initial microstructure used for thermomigration studies. The bright and dark phases are the $B$ - and $A$-rich phases, respectively. (b) Microstructure of a two-phase alloy annealed for $370 \mathrm{~h}$ in a temperature gradient, while the thermotransport effect was intentionally ignored during the simulation.

mation of $A$ - and $B$-rich single-phase layers at the hot and cold ends (i.e., demixing) for cases I, II, and III. However, in case IV, no such demixing was observed. It is noteworthy that the movement of elements is in opposite direction in case I and III. As we will discuss later, the above results suggest that even though the sign of the heat of transport term, $Q_{i}^{*}$ is positive, the direction of motion of elements and the final distribution of phases are dependent on the sign and magnitude of $M_{Q}$.

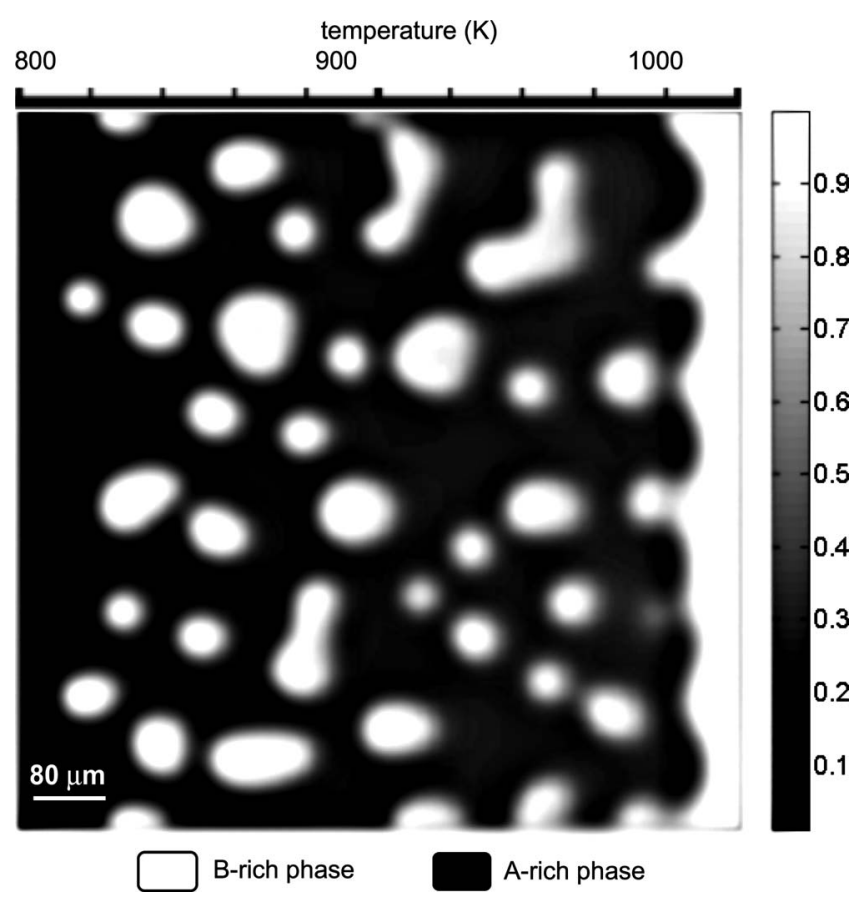

FIG. 4. Micrograph of the two-phase alloy obtained after an anneal for 370 $\mathrm{h}$ in a temperature gradient for case I where $Q_{A}^{*}=Q_{B}^{*}$ and $M_{Q}<0$. B atoms move toward the hot end forming a $B$-rich single-phase, while an $A$-rich phase forms at the cold end.

\section{DISCUSSION}

We have demonstrated that a phase field model can simulate the redistribution of constituent elements and phases when a binary alloy is subjected to a temperature gradient. The magnitude and direction of this redistribution, i.e., whether toward the hot end or the cold end of the specimen, is dependent on various factors and cannot be predicted simply by the phase diagram and Fickian diffusion. As a result, the flux equation has been modified to include the

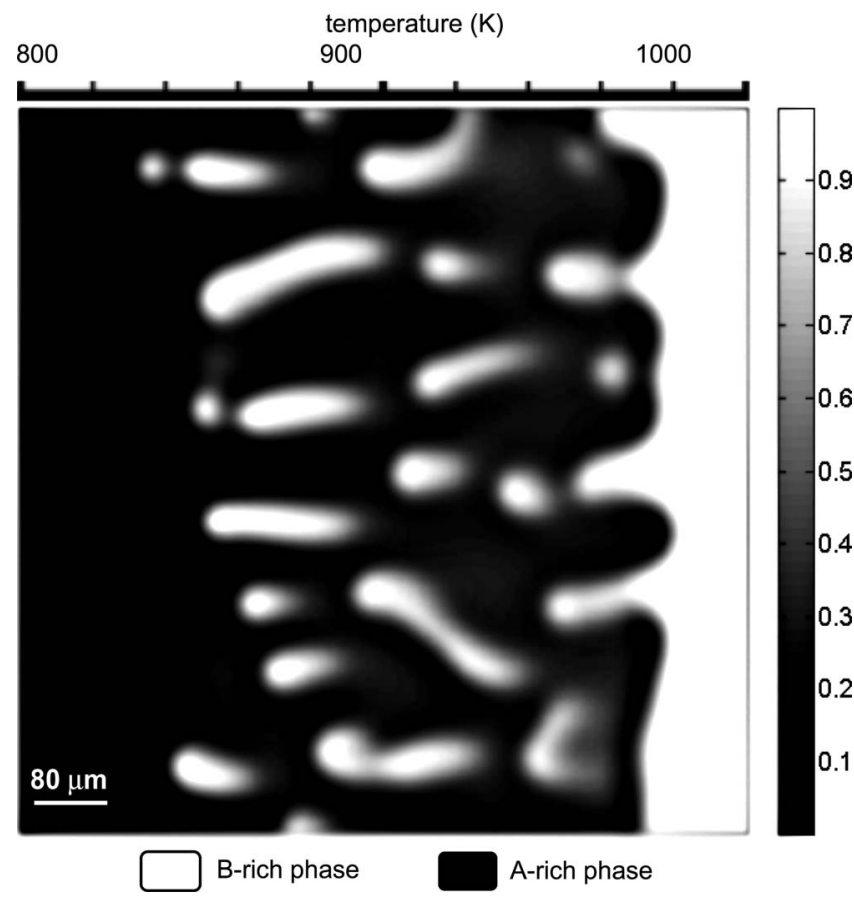

FIG. 5. Micrograph of the two-phase alloy obtained after an anneal for 370 $\mathrm{h}$ in a temperature gradient for case II where $Q_{B}^{*} \gg Q_{A}^{*}$ and $M_{Q}<0$. B atoms move toward the hot end forming a $B$-rich single-phase, while an $A$-rich phase forms at the cold end. 


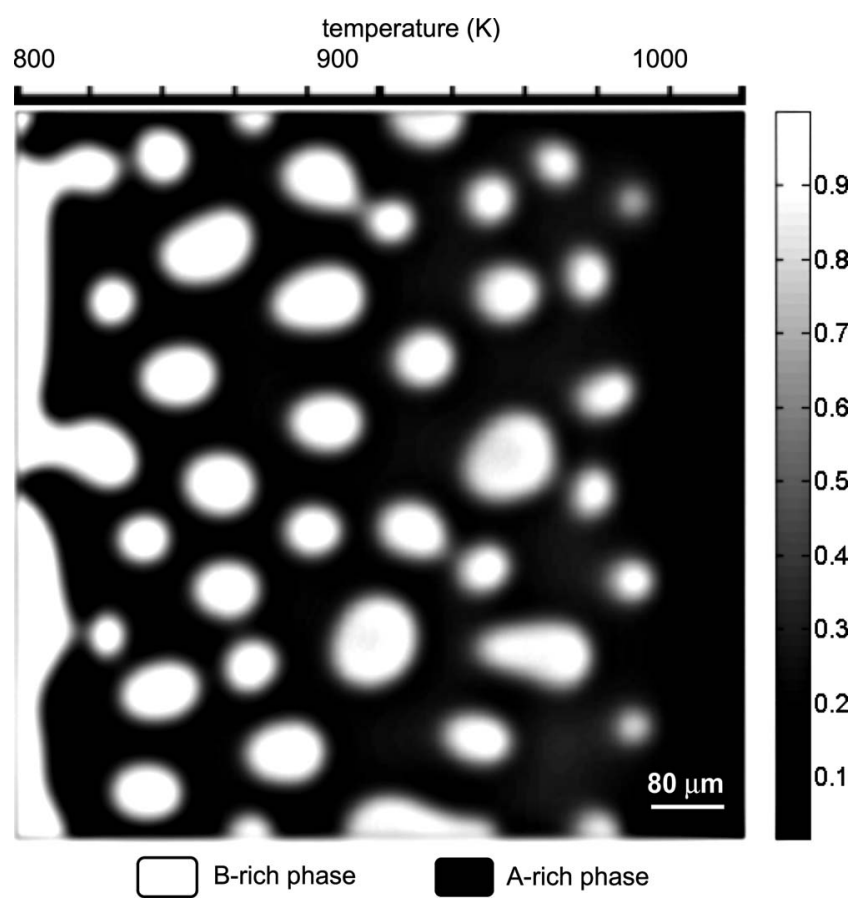

FIG. 6. Micrograph of the two-phase alloy obtained after an anneal for 370 $\mathrm{h}$ in a temperature gradient for case III where $Q_{B}^{*} \ll Q_{A}^{*}$ and $M_{Q}>0$. B atoms move toward the hot end forming a $B$-rich single-phase, while an $A$-rich phase forms at the cold end.

thermomigration effect. As described earlier, in case of a substitutional alloy where elements could have comparable mobilities, it is necessary that the heat of transport as well as mobilities of both the elements be considered in order to describe the flux of an element. The combination of these mobilities and heat of transport values determine the magni-

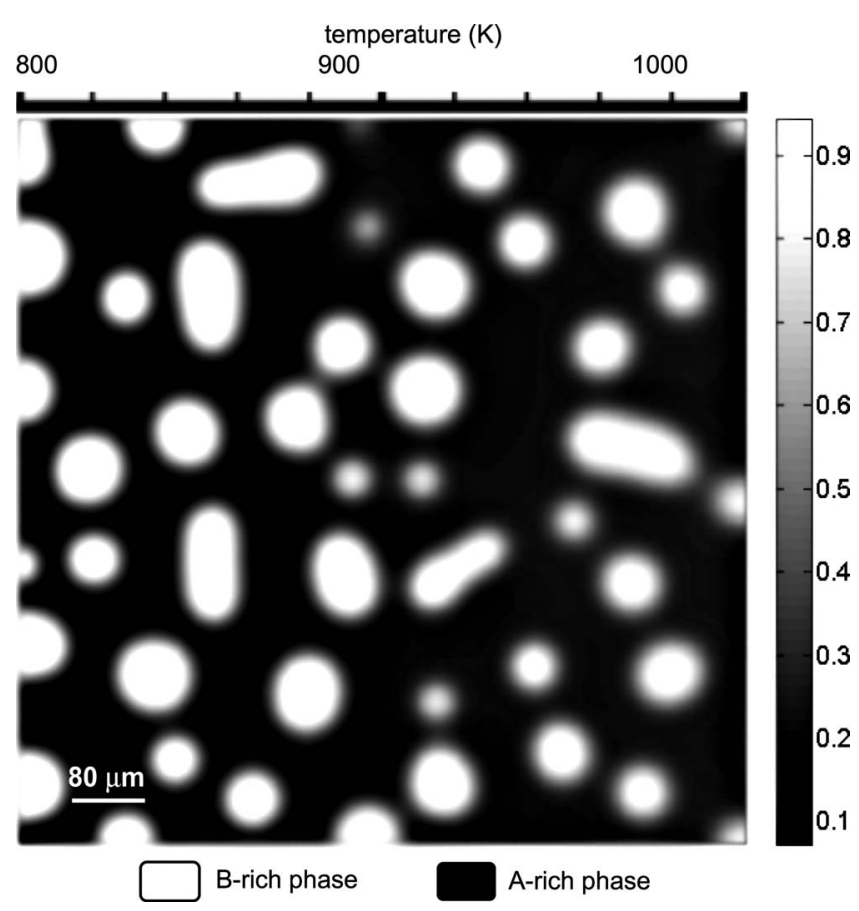

FIG. 7. Micrograph of the two-phase alloy obtained after being subjected to annealing for $370 \mathrm{~h}$ in a temperature gradient in case IV: $Q_{B}^{*}<Q_{A}^{*}, M_{Q}>0$, and $\left|M_{Q}\right|$ is small. The effect of thermomigration is less evident. tude and sign of mobility of thermotransport $M_{Q}$, which ultimately decides the direction of flow of an element.

For two-phase alloys, in case I, $Q_{A}^{*}=Q_{B}^{*}$ and $M_{Q}<0$. Since $M_{c}>0$, the contribution of the temperature gradient driven term to the flux of B in Eq. (17) is in the same direction as the term involving the concentration gradient. This causes the B atoms to flow toward the hot end. In case II, $Q_{B}^{*} \gg Q_{A}^{*}$ and $M_{Q}<0$, where $\left|M_{Q}\right|$ is very large. This produces a similar redistribution of the second phase, as that of case I, but the effect is much stronger. This is evident from the distinct flow pattern of $\mathrm{B}$ atoms toward the hot end throughout the system in Fig. 4(b).

Unlike cases I and II, $M_{Q}$ is positive in cases III and IV. This suggests that the contribution of temperature gradient to the flux of $\mathrm{B}$ atoms is opposite to the contribution by concentration gradient. This means that B atoms will flow toward the cold end, opposite to the direction predicted by the phase diagram of the alloy system. This is evident from the B-rich single-phase formed at the cold end of the specimen for case III where $\left|M_{Q}\right|$ is large as shown in Fig. 6. However, this redistribution is not appreciable for case IV since $\left|M_{Q}\right|$ is small and the contribution of temperature gradient to the total flux is negligible.

From Eq. (20) it can be deduced that for a binary solution with an immobile solvent, $M_{Q}$ is roughly equal to the product of atomic mobility and heat of transport of the solute, i.e., $\beta_{B} Q_{B}^{*}$. In this case, the flow direction of the solute can be predicted with a considerable degree of certainty from the knowledge of $Q_{B}^{*}$ only. The situation becomes more complex with the presence of a mobile solvent and the heat of transport of both solvent and solute contribute to the value of $M_{Q}$. An important consequence of the aforementioned, which is also exemplified by our results is that for a particular solvent, while different solutes can have heat of transport terms $\left(Q_{B}^{*}\right)$ of same sign, $M_{Q}$ may or may not have the same sign as that of $Q_{B}^{*}$ for all the solutes. Under such circumstances the prediction of the flow direction of solute atoms could be anomalous, and has been so experienced in many systems, e.g., $\mathrm{Co}, \mathrm{Au}, \mathrm{Ge}$, and $\mathrm{Ag}$ as solutes in $\mathrm{Cu} .^{5}$ This work demonstrates that the results of the present model are consistent with the experimental results on thermotransport in substitutional alloys. Being capable of taking into account the magnitude as well as the sign of individual mobility and heat of transport terms, this model can be of great help in predicting the flow behavior of solutes in a solvent under applied thermal gradient, when coupled with careful experimental studies.

Previous analytical and numerical models ${ }^{17-21}$ used to predict the thermotransport behavior in single- and twophase alloys derive heavily from the model first proposed by Shewmon $^{2}$ for binary alloys. Jaffe and Shewmon ${ }^{5}$ represented the drift velocity of solute and solvent in a binary substitutional alloy as

$$
V_{i}=-\frac{D_{i}}{R T}\left[R T \frac{\partial \ln c_{i}}{\partial x}+\frac{Q_{i}^{*}-\Delta H_{v i}}{T} \frac{d T}{d x}\right],
$$

where $\Delta H_{v i}$ is the molar enthalpy change in the lattice for forming a vacancy surrounded by the solute or solvent atoms. At steady state $V_{1}$ and $V_{2}$ may not be equal to zero, but 
$V_{1}$ is equal to $V_{2}$. However, apart from the assumption that local equilibrium concentration of vacancies is maintained at each point, drift-velocity model requires two other important assumptions, i.e., only the solute migrates in the alloy and local thermodynamic equilibrium between the solute in solution and in the second-phase particles is obtained in those regions where the solubility limit is exceeded. The present phase field model based on a continuum approach requires only the assumption of equilibrium vacancy concentration to be maintained locally, and does not demand the other two aforementioned assumptions to be made beforehand. This is due to the fact that the present model is derived using the actual thermodynamic description of the system. Furthermore, all the previous models are based on a 1D approach to solve the steady state problem, whereas a phase field model can describe the thermotransport behavior in any number of dimensions.

Using this model, the effect of temperature gradient on the composition and microstructure of any alloy can be predicted, provided the thermodynamics and diffusion kinetics that includes heat of transport terms are available. Thermodynamics and diffusion kinetics data for some alloy systems where thermotransport is important, such as U-alloys for nuclear fuel applications, Ni-alloys for gas turbine applications and $\mathrm{Pb}-\mathrm{Sn}$ alloys for interconnects applications are available in literature. The thermodynamic description for these systems can be obtained from a Calphad database, ${ }^{32-35}$ whereas the mobility data for $\mathrm{Ni}-\mathrm{Al}$ and $\mathrm{Pb}-\mathrm{Sn}$ can be obtained from literature. ${ }^{29,30}$ On the other hand, experimental data for heat of transport terms in these alloys are somewhat scarce. ${ }^{14,36,37}$ Simulations using various combinations of these unknown parameters are currently being carried out along with experimental determination of heat of transport terms.

\section{CONCLUSIONS}

It is well known that a temperature gradient can cause appreciable redistribution of elements and phases present in an alloy. A diffuse interface model was successfully devised to simulate and predict this redistribution phenomenon under applied temperature gradient. The results show that both atomic mobilities and heat of transport values of individual elements play a role in determining the magnitude and direction of flux that yields the development of concentration gradient. In certain cases the combination of these parameters can cause an element to flow opposite to the direction as determined by the phase diagram and Fick's first law. This model can be used to simulate real alloy systems by incorporating actual thermodynamic data and materials parameters for the system.

\section{ACKNOWLEDGMENTS}

Authors would like to thank the financial support from Idaho National Laboratory (Contract No. 00081977) under the operation of U.S. Department of Energy-Battelle Enegy Alliance, LLC (Contract No. DE-AC07-051D14517) and CAREER Award from National Science Foundation (Contract No. DMR-0238356). Technical guidance from Drs. Maria Okuniewski, Dennis D. Keiser, Jr., and Steven Hayes from Idaho National Laboratory is sincerely appreciated. Any opinion, findings, and conclusions or recommendations expressed in this manuscript are those of the authors and do not necessarily reflect the view of financial supports aforementioned.

${ }^{1}$ A. R. Allnatt and A. V. Chadwick, Chem. Rev. (Washington, D.C.) 67, 681 (1967).

${ }^{2}$ P. G. Shewmon, Trans. Metall. Soc. AIME 212, 642 (1958).

${ }^{3}$ P. G. Shewmon, J. Chem. Phys. 29, 1032 (1958).

${ }^{4}$ P. G. Shewmon, Acta Metall. 8, 605 (1960).

${ }^{5}$ D. Jaffe and P. G. Shewmon, Acta Metall. 12, 515 (1964).

${ }^{6}$ Y. H. Sohn, M. A. Dayananda, G. L. Hofman, R. V. Strain, and S. L. Hayes, J. Nucl. Mater. 279, 317 (2000).

${ }^{7}$ I. C. I. Okafor, O. N. Carlson, and D. M. Martin, Metall. Trans. A 13, 1713 (1982).

${ }^{8}$ M. Uz, D. K. Rehbein, and O. N. Carlson, Metall. Trans. A 17, 1955 (1986).

${ }^{9}$ S. C. Axtell and O. N. Carlson, Metall. Trans. A 21, 2141 (1990).

${ }^{10}$ J. G. Shaw and W. A. Oates, Metall. Trans. 2, 2127 (1971).

${ }^{11}$ C. J. Meechan and G. W. Lehman, J. Appl. Phys. 33, 634 (1962).

${ }^{12}$ W. Mock, Jr., Phys. Rev. 179, 663 (1969).

${ }^{13}$ A. T. Huang, A. M. Gusak, K. N. Tu, and Y. S. Lai, Appl. Phys. Lett. 88, 141911 (2006)

${ }^{14}$ F. Y. Ouyang, K. N. Tu, Y. S. Lai, and A. M. Gusak, Appl. Phys. Lett. 89, 221906 (2006).

${ }^{15}$ V. Tikare and E. A. Holm, J. Am. Ceram. Soc. 81, 480 (1998).

${ }^{16}$ V. A. Snyder, N. Akaiwa, J. Alkemper, and P. W. Voorhees, Metall. Mater. Trans. A 30, 2341 (1999).

${ }^{17}$ S. Van Vaerenbergh, S. R. Coriell, G. B. McFadden, B. T. Murray, and J. C. Legros, J. Cryst. Growth 147, 207 (1995).

${ }^{18}$ L. L. Zheng, D. J. Larson, Jr., and H. Zhang, J. Cryst. Growth 191, 243 (1998).

${ }^{19}$ G. P. Marino, Nucl. Sci. Eng. 49, 93 (1972).

${ }^{20}$ D. Yang, B. Y. Wu, Y. C. Chan, and K. N. Tu, J. Appl. Phys. 102, 043502 (2007).

${ }^{21}$ G. L. Hofman, S. L. Hayes, and M. C. Petri, J. Nucl. Mater. 227, 277 (1996).

${ }^{22}$ J. W. Cahn and J. E. Hilliard, J. Chem. Phys. 28, 258 (1958).

${ }^{23}$ L. Q. Chen, Annu. Rev. Mater. Res. 32, 113 (2002).

${ }^{24} \mathrm{~S}$. R. de Groot, Thermodynamics of Irreversible Processes (NorthHolland, Amsterdam, 1952).

${ }^{25}$ R. E. Howard and A. B. Lidiard, Rep. Prog. Phys. 27, 161 (1964).

${ }^{26}$ J. Philibert, Atom Movements, Diffusion and Mass Transport in Solids, Les Éditions de Physique (Courtaboeuf, Les Ulies, France, 1991), p. 326.

${ }^{27}$ A. R. Allnatt and A. B. Lidiard, Atomic Transport in Solids (Cambridge University Press, Cambridge, 1993), pp. 165-191.

${ }^{28}$ P. G. Shewmon, Diffusion in Solids (McGraw-Hill, New York, 1963), pp. 134-135.

${ }^{29}$ A. Engstrom and J. Agren, Z. Metallkd. 87, 92 (1996).

${ }^{30}$ G. Ghosh and Z. K. Liu, J. Electron. Mater. 27, 1362 (1998).

${ }^{31}$ J. E. Guyer, D. Wheeler, and J. A. Warren, Comput. Sci. Eng. 11, 6 (2009), http://www.ctcms.nist.gov/fipy (updated 6 February 2009).

${ }^{32}$ A. T. Dinsdale, Calphad 15, 317 (1991).

${ }^{33}$ P. Y. Chevalier, E. Fisher, and B. Cheynet, Calphad 28, 15 (2004).

${ }^{34}$ I. Ansara, N. Dupin, H. L. Lukas, and B. Sundman, J. Alloys Compd. 247, 20 (1997).

${ }^{35}$ H. Ohtani, K. Okuda, and K. Ishida, J. Phase Equilib. 16, 416 (1995).

${ }^{36}$ J. F. D' Amico and H. B. Huntington, J. Phys. Chem. Solids 30, 1697 (1969).

${ }^{37}$ D. R. Campbell and H. B. Huntington, Phys. Rev. 179, 601 (1969). 